\title{
VERTICAL TRACK STIFFNESS EFFECT ON DYNAMIC BEHAVIOUR OF TRACK STRUCTURE
}

The dynamic modelling of railway track response and of the interaction of vehicle and track at low and mid frequencies that are significant for the track deterioration, is presented. The response of the track subjected to a moving vehicle is simulated for a stationary randomly distributed ballast stiffness with a standard uniform distribution and a normal distribution. The response of the track components is solved by the Finite Element Method. Monte Carlo simulation was applied to estimate the dynamic track response. The dynamic amplification resulting from the simulated passage of the locomotive of the type $E 499$ ( $85 t$ ) is presented.

\section{Introduction}

The dynamic response of a railway track subjected to moving vehicles in service conditions generally has a random character. Major sources of dynamic effects on the vertical response of track - isolated and periodic irregularities on the rail surface, repetitive effect of the sleeper spacing, random variations of longitudinal profile of the track, etc., are frequently investigated. The less attention is paid to the influence of the vertical track stiffness on the response of track components and on the evaluation of forces transmitted by the track components.

The model presented in this paper, developed at the Department of Mechanics [6], was addressed to the study of dynamic behaviour of track structure, especially the evaluation of the dynamic coefficient for the track components. The dynamic behaviour and the response of track components (rails, sleepers, the ballast) due to variability in the vertical stiffness of rail supports are presented in this paper. Time domain technique has been used to study these dynamic effects. The approach can be helpful in clarifying the influence of vehicle speed on the dynamic response of the track components.

Using the computer program Interaction [6] the Monte Carlo Simulations were applied. With the prescribed set of input values for system parameters, the simulation process yielded a specific measure of response.

\section{Outline of solution techniques}

The theoretical model of the rail as a beam on continuous elastic foundation loaded by a vertical concentrated force P moving along at a constant speed $\mathrm{c}$ introduced by Timoshenko provides a basis for the track design procedure and the stress analysis of the railway track components. The equation of motion of the rail for this case is [1, 5], see Fig. 1:

$$
\begin{aligned}
& E I \frac{\partial^{4} w\left(x^{\prime}, t\right)}{\partial x^{\prime 4}}+\mu_{x} \frac{\partial^{2} w\left(x^{\prime}, t\right)}{\partial x^{\prime 2}}+2 \mu_{x} \omega_{b} \frac{\partial w\left(x^{\prime}, t\right)}{\partial t}+ \\
& +\kappa_{z} \cdot w\left(x^{\prime}, t\right)=P \cdot \delta\left(x^{\prime}\right)
\end{aligned}
$$

where: $w\left(x^{\prime}, t\right)$ is the vertical motion (dynamic deflection) of the rail in the stationary co-ordinates $\left(x^{\prime}, t\right)$

$C_{b}=2 \mu_{x} \omega_{b}$ is a damping coefficient of structure,

$\omega_{b} \quad\left[\mathrm{~s}^{-1}\right]$ is a angular damping frequency,

$\kappa_{z}\left[\mathrm{Nm}^{-1}\right]$ is the stiffness per length of rail support,

$E I \quad$ is a flexural rigidity of rail,

$\mu_{x} \quad$ is the mass of rail per unit length,

$c \quad$ is the speed of moving force $P$.

The equation of motion of the rail in the moving co-ordinates $(x, t)$ has the form, [5]:

$$
\begin{aligned}
& E I \frac{\partial^{4} w(x, t)}{\partial x^{4}}+\mu_{x}\left[\frac{\partial^{2} w(x, t)}{\partial t^{2}}-2 c \frac{\partial^{2} w(x, t)}{\partial x \partial t}+c^{2} \frac{\partial^{2} w(x, t)}{\partial x^{2}}\right]+ \\
& +2 \mu_{x} \omega_{b}\left[\frac{\partial w(x, t)}{\partial t}-c \frac{\partial w(x, t)}{\partial x}\right]+\kappa_{z} \cdot w(x, t)= \\
& =P \cdot \delta(x-c t)
\end{aligned}
$$

where: $w(x, t)$ is the vertical displacement function (dynamic deflection) of the rail in the moving co-ordinates $(x, t)$.

A number of other theoretical models $[2,5]$ have been developed for the track structure behaviour under vertical loads which include either separate components of the track structure (rails, fasteners, sleepers and subgrade) or total characteristics of the track structure, see Fig. 2.

The differential equation (2) of the problem can be solved either in the frequency or in the time domain, [2]. But for the sto-

\footnotetext{
* Milan Moravčík

Department of Mechanics, Faculty of Civil Engineering, University of Žilina, Komenského 52, 01001 Žilina, Slovak Rep.,

E-mail: mimo@fstav.utc.sk
} 


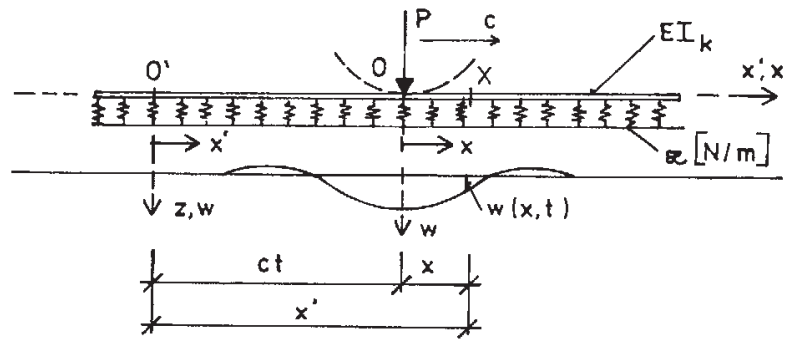

Fig. 1 Rail as a beam on continuously elastic foundation

chastic modeling of the track stiffness $\kappa=\{\kappa(\mathrm{x})\}$ solving of Eq. 2 brings about serious troubles. At present, the Finite Element Approach (FEA) is adopted for solution of these effects. Fig. 2 shows the used physical modeling of the track components in vertical direction corresponding to the FEA application. The mechanical properties of the track components are modeled by a set of springs and dampers in one or two layers. The characteristics of
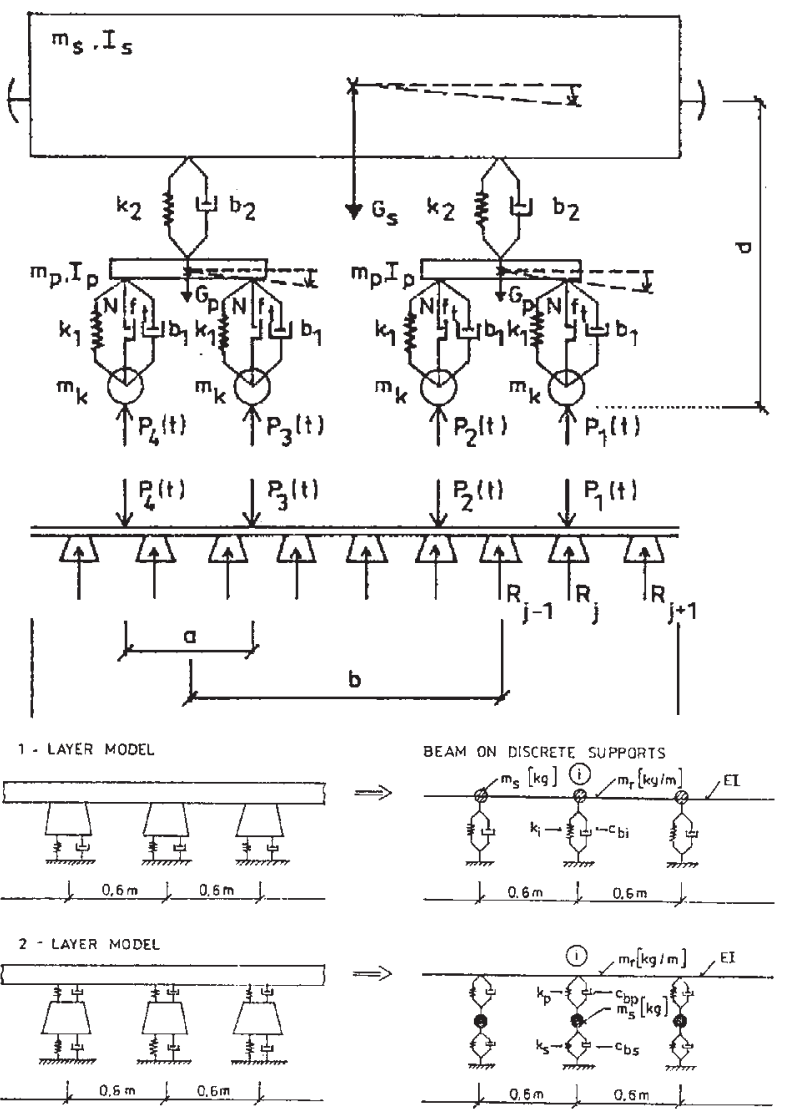

3- LAYER DISCRETE MODEL

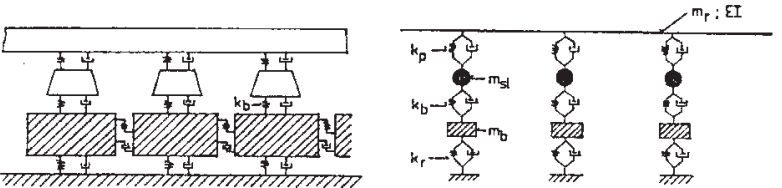

Fig. 2 Theoretical models of track structure in vertical direction springs and dampers can be determined by the laboratory load tests of these components or by the field measurements in typical track conditions.

\section{Approach}

The track behaviour under dynamic loading conditions is reproduced by an interactive dynamic model with three model components:

- the dynamic model of vehicle,

- the linear track model,

- the stiffness of discrete rail supports.

A plain mathematical model is adopted aiming at the vertical vehicle-track interaction problem for low and medium frequency ranges, say for the frequency bandwidth of $0-200 \mathrm{~Hz}$. The Finite Element Method is used for modeling of the track and the Composite Element Method is used for the modeling of a vehicle. The vehicle-track/interaction model is schematically shown in Fig. 3. This model allows to describe the dynamic behaviour of the two subsystems - the vehicle and the track.
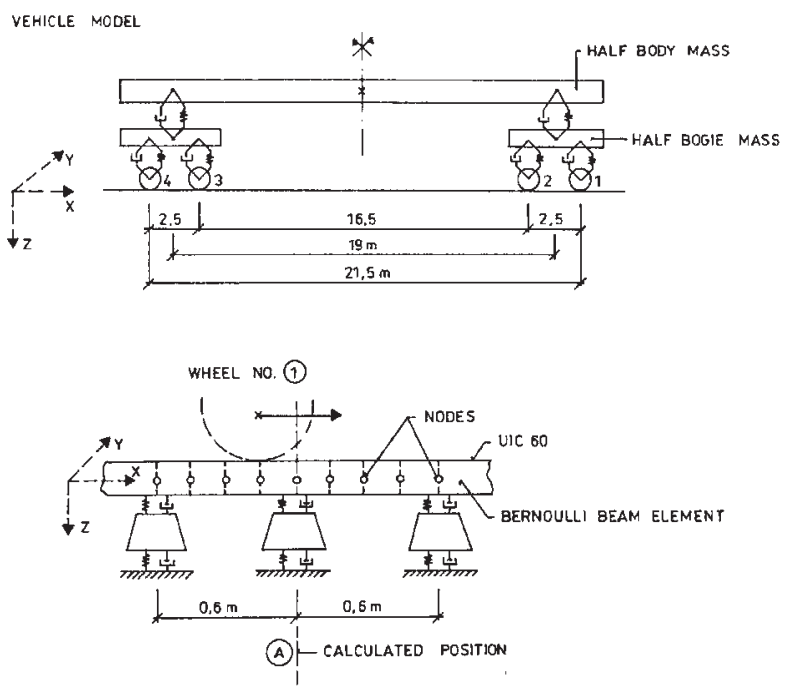

Fig. 3 Scheme of the vehicle-track/interaction model

The rail with its elastic properties is modelled as an EulerBernoulli beam using the finite element approach. The nodes of the finite elements lie above each sleeper and have two degrees of freedom - vertical translation and pitch rotation. The four elements belong to one sleeper bay. The cubic polynomial functions are used to describe the element displacement. The sleepers are considered as rigid bodies. The stiffness and damping of railpads, the ballast and the subgrade are simulated for each sleeper. The resulting equations of motion of the track structure are:

$$
[M]\{\ddot{U}\}+[C]\{\dot{U}\}+[K]\{U\}=\{P\}
$$


where $[M],[C]$ and $[K]$ are the mass, damping and stiffness matrices of the track structure respectively, and $\{P\}$ is the nodal load vector. $\{U\}$ is the nodal vertical displacement vector.

The vehicle is modelled by the Composite Element Method as a system described by three quantities: its mass or inherent properties, its internal force elements (springs and dampers) and the generalised coordinates. The equation corresponding of motion is given by

$$
[m][\ddot{u}\}+[c]\{\dot{u}\}+[k]\{u\}=\{F\}
$$

where: $[m],[c]$ and $[k]$ are the mass, damping and stiffness matrices of the vehicle system respectively, and $\{F\}$ is the nodal load vector. $\{u\}$ is the nodal vertical displacement vector.

Numerical algorithms consisting of finite-element procedures to model the track structure and time -step integration to calculate the response have been used, [3]. The solution algorithm for the time-domain technique is illustrated in Fig. 4.

TIME DOMAIN SOLUTION ALGORITHM

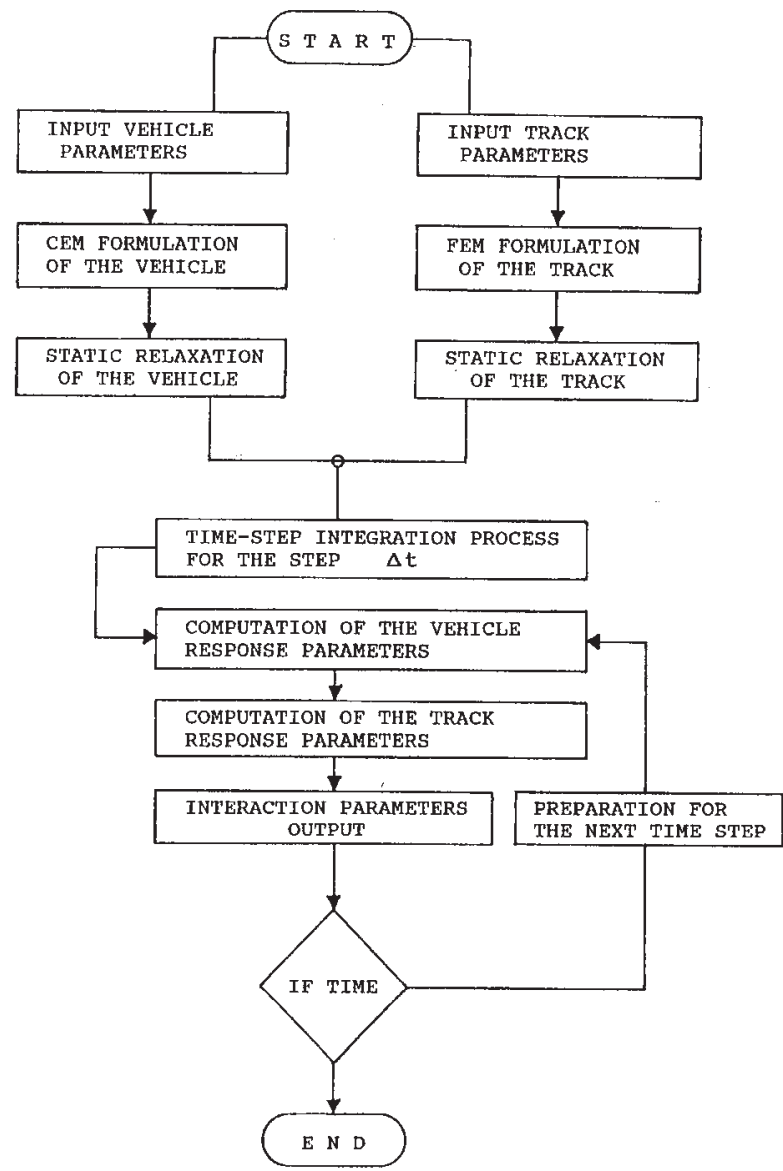

Fig. 4 Time domain solution algorithm

\subsection{Variable vertical track stiffness modeling}

In order to examine the effect of variable track stiffness conditions, the five characteristic vertical ballast stiffness ${ }^{(i)} k_{b}, i=1 \div 5$, of the track were taken to model low, medium, and high track stiffness conditions. The mean values of the vertical ballast stiffness and the vertical pad stiffness $k_{f}$ are shown in Tab. 1 .

The stiffness levels $(i)$ in Tab. 1 are considered the basic mean values for the simulation of discrete random variables ${ }^{(i)} k_{b, j}$ of the vertical track stiffness $k_{b}$ for the study of influence of the track stiffness on the response. In this study only the variability of vertical track stiffness is considered to be a random input quantity and the next input parameters are considered the deterministic ones.

Mean values of the modelled vertical stiffness of track Tab. 1

\begin{tabular}{|c|c|c|c|c|}
\hline $\begin{array}{c}\text { Track } \\
\text { type } \\
(\mathrm{i})\end{array}$ & $\begin{array}{c}\text { Level of } \\
\text { Support }\end{array}$ & $\begin{array}{c}\text { Vertical } \\
\text { Ballast Stiffness } \\
{ }^{(i)} \overline{k_{b}}[\mathrm{~N} / \mathrm{m}]\end{array}$ & \multicolumn{2}{|c|}{$\begin{array}{c}\text { Vertical } \\
\text { Pad Stiffness } \\
\overline{k_{f}}[\mathrm{~N} / \mathrm{m}]\end{array}$} \\
\hline A & low & $40.10^{6}$ & 1 & $\begin{array}{c}150.10^{6} \\
2\end{array}$ \\
\hline B & medium & $80.10^{6}$ & 1 & $\begin{array}{c}150.10^{6} \\
60.10^{6}\end{array}$ \\
\hline C & medium & $120.10^{6}$ & 1 & $\begin{array}{c}150.10^{6} \\
2\end{array}$ \\
\hline D & high & $220.10^{6}$ & 1 & $150.10^{6}$ \\
\hline \multirow{2}{*}{ E } & very high & $480.10^{6}$ & 2 & $60.10^{6}$ \\
\hline
\end{tabular}

Two models for the random discrete rail stiffness supports were considered:

1/ The stationary randomly distributed stiffness of the discrete rail supports due to the random ballast stiffness $k_{b, j}$ with:

- the standard uniform distribution,

- the normal distribution.

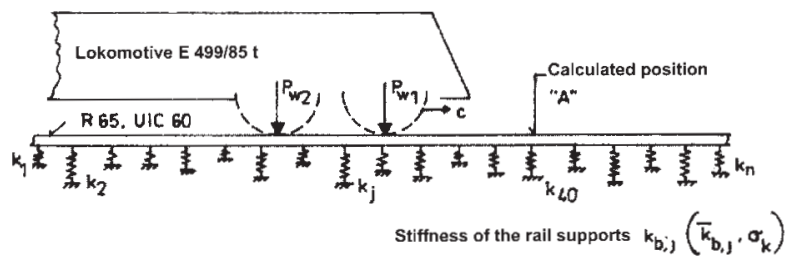

Fig. 5 Stationary randomly distributed vertical stiffness of the rail supports

The relative vertical ballast stiffness $\kappa_{b, j}$ can be applied on each stiffness level $(i)$

$$
k_{b, j}=\frac{{ }^{(i)} k_{b, j}}{{ }^{(i)} \bar{k}_{b}}
$$


where: ${ }^{(i)} \bar{k}_{b}$ is the mean value of the ballast stiffness on the level (i).

The relative ballast stiffness $k_{b, j}$ has been generated for the simulation studies and Monte -Carlo analyses, $k_{b, j}=k_{b, j, g e n}$, and the calculation has been carried out for:

a) the uniform distribution $k_{b, j}$

The relative ballast stiffness ${ }^{(i)} k_{b, j}$ kwas considered from the interval:

- Case 1: $0,75<{ }^{(i)} k_{b, j, \text { gen }}<1,25$, with the mean $\bar{k}_{\text {gen }}=1,0$ and the standard deviation $\sigma_{\kappa, \text { gen }}=0,145$.

- Case 2: $0,5<{ }^{(i)} k_{b, j, g e n}<1,5$, with the mean $\bar{k}_{\text {gen }}=1,0$ and the standard deviation $\sigma_{\kappa, \text { gen }}=0,288$.

b) For the normal distribution $k_{b, j}$

The relative ballast stiffness ${ }^{(i)} k_{b, j}$ were considered with parameters:

Case 1: $\bar{k}_{\text {gen }}=1,0 ; \sigma_{\kappa, g e n}=0,316, \sigma_{\kappa, \text { gen }}^{2}=0,1$,

and coefficient of variation $V_{\kappa}=\frac{\sigma_{\kappa, g e n}}{\bar{k}_{g e n}}=0,316$.

Case 2: $\bar{k}_{\text {gen }}=1,0 ; \sigma_{\kappa, \text { gen }}=0,447, \sigma_{\kappa, \text { gen }}^{2}=0,2$,

and coefficient of variation $V_{\kappa}=\frac{\sigma_{\kappa, g e n}}{\bar{k}_{\text {gen }}}=0,447$.

The corresponding real ballast stiffness ${ }^{(i)} k_{b, j}$ is:

$$
{ }^{(i)} k_{b, j}={ }^{(i)} \kappa_{b, j, \text { gen }} \cdot{ }^{(i)} \bar{k}_{b} \text {. }
$$

\section{Results of numerical simulation}

Simulation results include the chosen amplitudes of the dynamic track response $Y_{d y n}=(w, P, M .$.$) : the rail deflection w$ in the position above the sleeper "A", the sleeper - ballast force $P_{s-b}$ under the sleeper " $\mathrm{A}$ ", and the rail bending moment $M$, etc.

The simulation was made for the locomotive Skoda E 499 ( $85 \mathrm{t}$ ) operating speeds of 20 and $50 \mathrm{~m} / \mathrm{s}$. The results of dynamic response $Y_{d y n}$ are compared with the static response $Y_{s t}$ for the modelled track stiffness type. In this paper are presented only some results for the track of the type B, see Table. 1. 2000 simulation runs were performed for both static and tested speeds of $20 \mathrm{~m} / \mathrm{s}$ and $50 \mathrm{~m} / \mathrm{s}$.

- Track stiffness B1 - the static response

Characteristics of response for the static rail deflection $w$, the sleeper ballast force $P_{s-b}$, for the uniform distribution of vertical support stiffness $k_{b, j}$ and for the Gaussian distribution of vertical support stiffness $k_{b, j}$ are presented in histograms in Fig. 6.

- Track stiffness B1 - the dynamic response

Characteristics of response for the dynamic rail deflection $w$ and the sleeper ballast force $P_{s-b}$, for the Uniform distribution of ver-
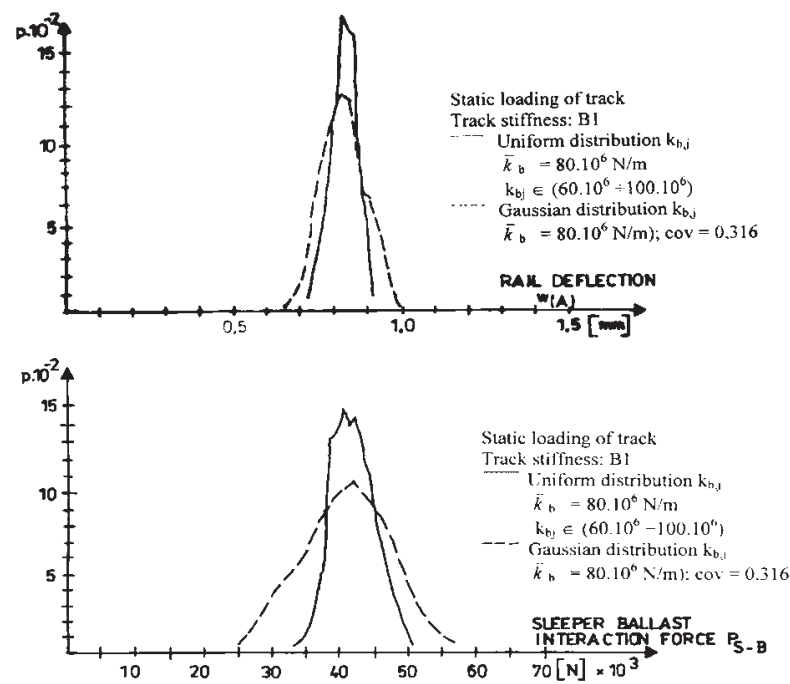

Fig. 6 Histograms of rail deflections $w$ and the sleeper ballast force $P_{b-s}$, for the Uniform and the Gaussian distribution of the vertical súpport stiffness $k_{b j}$, - the static case (the locomotive speed $c=0$ )

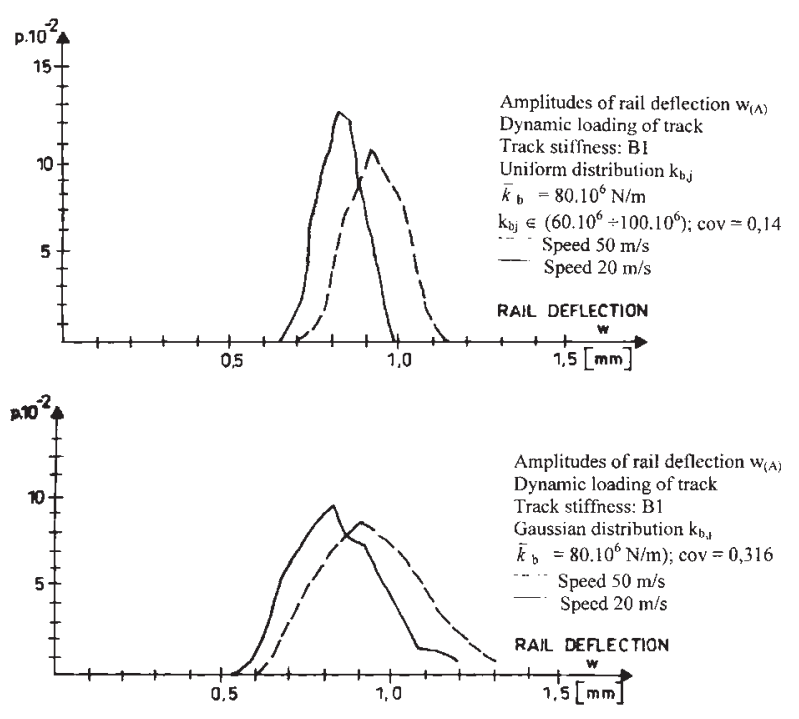

Fig. 7 Histograms of dynamic amplitudes of the rail deflection $w$ for the Uniform and the Gaussian distribution vertical support stiffness $k_{b j}$, for the locomotive speed $20 \mathrm{~m} / \mathrm{s}$ and $50 \mathrm{~m} / \mathrm{s}$

tical support stiffness $k_{b, j}$, and for the Gaussian distribution $k_{b, j}$, are presented on histograms in Fig. 7 and Fig. 8.

Track stiffness B1 - Comparison results of the static and dynamic response for the uniform and Gaussian distribution of the vertical supports stiffness $k_{b j}$, see Fig. $9 \div$ Fig. 10 .

\section{Dynamic coefficient}

A moving vehicle on a track with stochastic rigidity of the substructure in the vertical direction generates deflections and stresses 

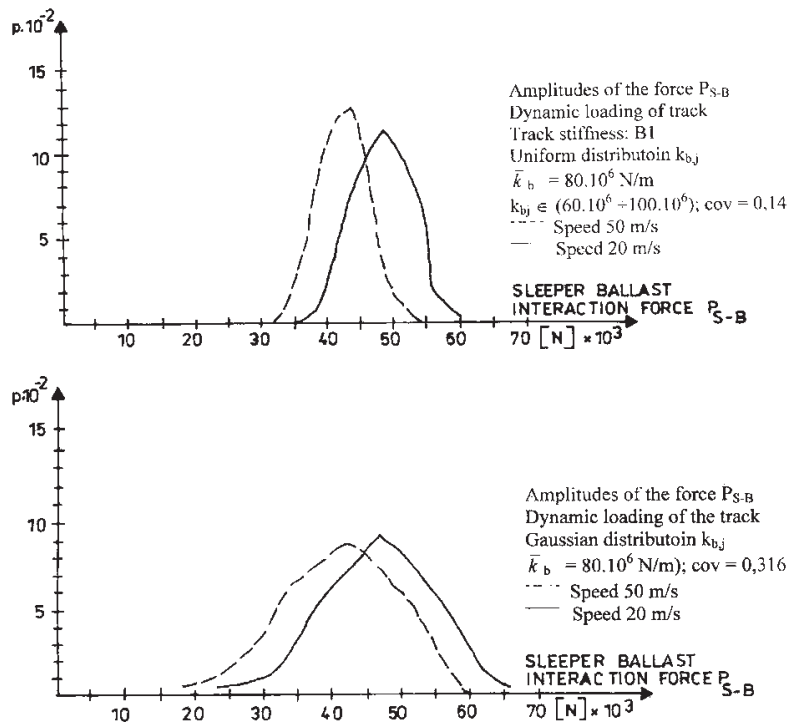

Fig. 8 Histograms of dynamic amplitudes of the sleeper-ballast force $P_{s-b}$ for the uniform and Gaussian distribution vertical support stiffness $k_{b j}$, for the locomotive speed $20 \mathrm{~m} / \mathrm{s}$ and $50 \mathrm{~m} / \mathrm{s}$
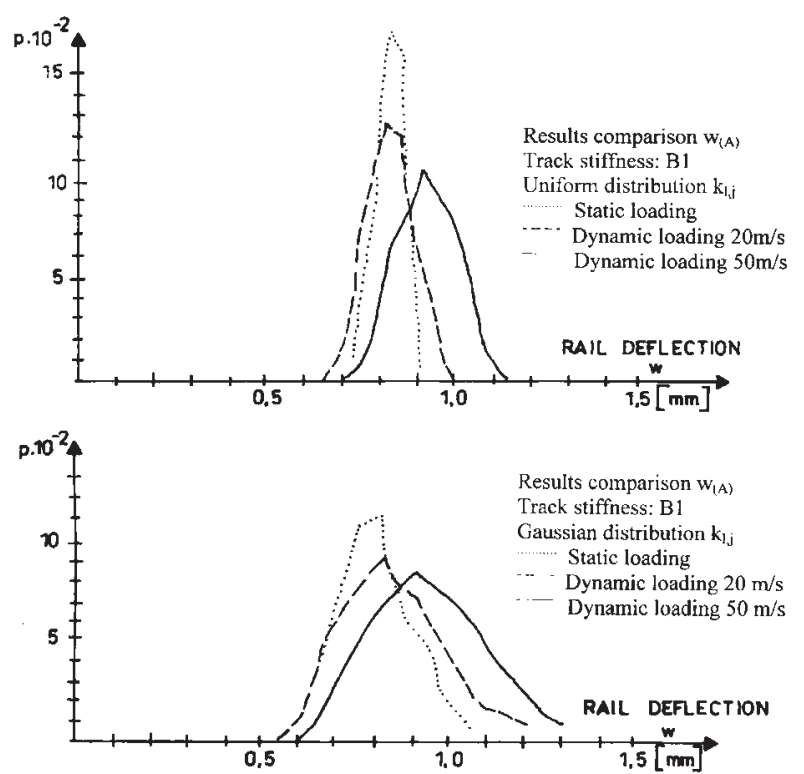

Fig. 9 Histograms of the rail deflection $w$ - comparison the static and dynamic response for the Uniform distribution and Gaussian distribution of the stiffness $k_{b, j}$

in the track structure that are generally greater than those caused by the same vehicle load applied statically or moving on the track with constant rigidity of the substructure. The dynamic amplification $\delta$ resulting from the passage of the vehicle over the track section with the simulated stiffness of supports can be defined as the ratio of the maximum dynamic amplitude of a quantity $Y_{d y n(r a n d)}$ (rail deflection $w$, bending moment $M$, etc.) to the static ampli-
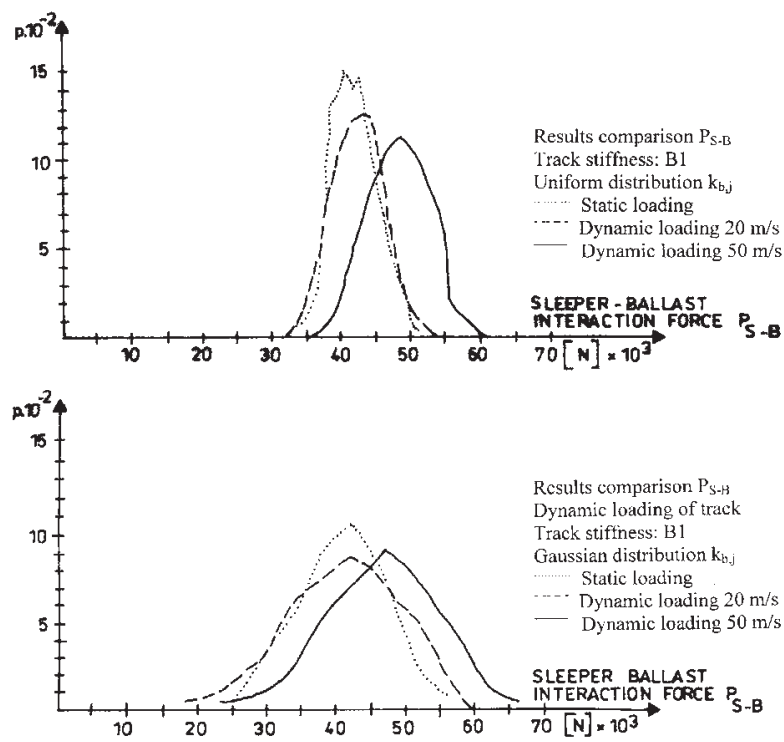

Fig. 10 Histograms of the sleeper-ballast force amplitude $P_{s-b}$ comparison the static and dynamic response for the Uniform distribution and Gaussian distribution of the stiffness $k_{b, j}$

tude of a quantity $Y_{s t(c o n s)}$, for the tested type of the track $(i)$ with constant stiffness of supports $k_{b, j}$.

$$
\delta=\frac{Y_{d y n}(\text { rand })}{Y_{s t(\text { cons })}}
$$

The passage of each wheelset induces a peak of the observed quantity $Y_{d y n}$ and the results may be treated statistically as shown in histograms in previous figures. Thus, the histograms may be exploited for the statistically expected values of the dynamic coefficient for the rail deflection, bending moments, etc. The dynamic coefficients $\delta$ of the chosen quantities for the track $\mathrm{B} 1$, for constant stiffness of supports, are presented in Tab. 2 .

Dynamic coefficient $\delta$ for the constant

Tab. 2 stiffness of support, $k_{b, j}=$ const.

\begin{tabular}{|c|c|c|c|c|c|}
\hline \multirow{3}{*}{$\begin{array}{c}\text { Track } \\
\text { B1 }\end{array}$} & \multicolumn{3}{|c|}{ Response } & \multirow{2}{*}{\multicolumn{2}{|c|}{$\begin{array}{c}\text { Dynamic coefficient } \\
\delta=Y_{d y n} / Y_{s t}\end{array}$}} \\
\hline & \multirow[t]{2}{*}{$Y_{s t}$} & \multicolumn{2}{|c|}{$Y_{d y n}$} & & \\
\hline & & $20 \mathrm{~m} / \mathrm{s}$ & $50 \mathrm{~m} / \mathrm{s}$ & $20 \mathrm{~m} / \mathrm{s}$ & $50 \mathrm{~m} / \mathrm{s}$ \\
\hline $\begin{array}{c}\text { Rail } \\
\text { Deflection } \\
Y=v[\mathrm{~mm}]\end{array}$ & 0.796 & 0.821 & 0.908 & 1.03 & 1.14 \\
\hline $\begin{array}{c}\begin{array}{c}\text { Sleeper- } \\
\text { ballast }\end{array} \\
\text { Force } \\
Y=P[\mathrm{kN}]\end{array}$ & 41.5 & 42.9 & 47.7 & 1.03 & 1.15 \\
\hline
\end{tabular}

Using the mean values of the response quantities $\bar{Y}$ and the standard deviation $\sigma_{Y}$, the dynamic coefficient $\delta$ may be determined, see Tables $3 \div 6$.

The dynamic coefficient $\delta$ for the constant stiffness of supports, see Tab. 3, is small for the low vehicle speed and increases with the vehicle speed. The effect of stochastic rail support stiff- 
Dynamic coefficient $\delta$ for the Uniform distribution of support stiffness $k_{b, j}$, case 1

Tab. 3

\begin{tabular}{|c|c|c|c|c|c|c|}
\hline \multirow{2}{*}{$\begin{array}{c}\text { Track } \\
\text { B1 }\end{array}$} & \multicolumn{4}{|c|}{$k_{b, j} \in<60.10^{6}-100.10^{6} \mathrm{~N} / \mathrm{m}>$} \\
\cline { 2 - 7 } & \multicolumn{2}{|c|}{$\delta=\bar{Y}_{d y n} / Y_{s t}$} & \multicolumn{2}{c|}{$\delta=\left(\bar{Y}_{d y n}+\sigma_{y}\right) / Y_{s t}$} & \multicolumn{2}{c|}{$\delta=\left(\bar{Y}_{d y n}+2 \sigma_{y}\right) / Y_{s t}$} \\
\cline { 2 - 7 } & $20 \mathrm{~m} / \mathrm{s}$ & $50 \mathrm{~m} / \mathrm{s}$ & $20 \mathrm{~m} / \mathrm{s}$ & $50 \mathrm{~m} / \mathrm{s}$ & $20 \mathrm{~m} / \mathrm{s}$ & 1.33 \\
\hline Rail Deflection $Y=w$ & 1.03 & 1.15 & 1.18 & 1.33 & 1.51 & 1.50 \\
\hline Sleeper-ballast Force $Y=P_{s-b}$ & 1.01 & 1.14 & 1.17 & 1.5 & 1.32 & 1.51 \\
\hline
\end{tabular}

Dynamic coefficient $\delta$ for the Uniform distribution of support stiffness $k_{b, j}$, case 2

\begin{tabular}{|c|c|c|c|c|c|c|}
\hline \multirow{2}{*}{$\begin{array}{c}\text { Track } \\
\text { B1 }\end{array}$} & \multicolumn{4}{|c|}{$k_{b, j} \in<60.10^{6}-120.10^{6} \mathrm{~N} / \mathrm{m}>$} \\
\cline { 2 - 7 } & \multicolumn{2}{|c|}{$\delta=\bar{Y}_{d y n} / Y_{s t}$} & \multicolumn{2}{c|}{$\delta=\left(\bar{Y}_{d y n}+\sigma_{y}\right) / Y_{s t}$} & \multicolumn{2}{c|}{$\delta=\left(\bar{Y}_{d y n}+2 \sigma_{y}\right) / Y_{s t}$} \\
\cline { 2 - 7 } & $20 \mathrm{~m} / \mathrm{s}$ & $50 \mathrm{~m} / \mathrm{s}$ & $20 \mathrm{~m} / \mathrm{s}$ & $50 \mathrm{~m} / \mathrm{s}$ & $20 \mathrm{~m} / \mathrm{s}$ & 1.44 \\
\hline Rail Deflection $Y=w$ & 1.06 & 1.20 & 1.25 & 1.43 & 1.39 & 1.67 \\
\hline Sleeper-ballast Force $Y=P_{b-s}$ & 1.00 & 1.14 & 1.19 & 1.38 & 1.39 \\
\hline
\end{tabular}

Dynamic coefficient $\delta$ for the Gaussian distribution of the support stiffness $k_{b, j}$, case 1

Tab. 5

\begin{tabular}{|c|c|c|c|c|c|c|}
\hline \multirow{2}{*}{$\begin{array}{c}\text { Track } \\
\text { B1 }\end{array}$} & \multicolumn{4}{|c|}{$\bar{k}_{b}=80.10^{6} \mathrm{~N} / \mathrm{m} ; V_{k}=0.316$} \\
\cline { 2 - 7 } & \multicolumn{2}{|c|}{$\delta=\bar{Y}_{d y n} / Y_{s t}$} & \multicolumn{2}{c|}{$\delta=\left(\bar{Y}_{d y n}+\sigma_{y}\right) / Y_{s t}$} & \multicolumn{2}{c|}{$\delta=\left(\bar{Y}_{d y n}+2 \sigma_{y}\right) / Y_{s t}$} \\
\cline { 2 - 7 } & $20 \mathrm{~m} / \mathrm{s}$ & $50 \mathrm{~m} / \mathrm{s}$ & $20 \mathrm{~m} / \mathrm{s}$ & $50 \mathrm{~m} / \mathrm{s}$ & $20 \mathrm{~m} / \mathrm{s}$ & 1.51 \\
\hline Rail Deflection $Y=w$ & 1.08 & 1.21 & 1.30 & 1.47 & 1.5 & 1.69 \\
\hline Sleeper-ballast Force $Y=P_{s-b}$ & 1.00 & 1.14 & 1.23 & 1.41 & 1.47 & \\
\hline
\end{tabular}

Dynamic coefficient $\delta$ for the Gaussian distribution of the support stiffness $k_{b, j}$, case 2

\begin{tabular}{|c|c|c|c|c|c|c|}
\hline \multirow{2}{*}{$\begin{array}{c}\text { Track } \\
\text { B1 }\end{array}$} & \multicolumn{4}{|c|}{$\bar{k}_{b}=80.10^{6} \mathrm{~N} / \mathrm{m} ; V_{k}=0.316$} \\
\cline { 2 - 7 } & \multicolumn{2}{|c|}{$\delta=\bar{Y}_{d y n} / Y_{s t}$} & \multicolumn{2}{c|}{$\delta=\left(\bar{Y}_{d y n}+\sigma_{y}\right) / Y_{s t}$} & \multicolumn{2}{c|}{$\delta=\left(\bar{Y}_{d y n}+2 \sigma_{y}\right) / Y_{s t}$} \\
\cline { 2 - 7 } & $20 \mathrm{~m} / \mathrm{s}$ & $50 \mathrm{~m} / \mathrm{s}$ & $20 \mathrm{~m} / \mathrm{s}$ & $50 \mathrm{~m} / \mathrm{s}$ & $20 \mathrm{~m} / \mathrm{s}$ & 1.74 \\
\hline Rail Deflection $Y=w$ & 1.14 & 1.31 & 1.44 & 1.67 & 1.58 & 1.90 \\
\hline Sleeper-ballast Force $Y=P_{s-b}$ & 1.00 & 1.14 & 1.29 & 1.52 & 2.50 \\
\hline
\end{tabular}

ness can not be ignored, in particular for the higher vehicle speed, see Tab. $3 \div 6$. The results obtained confirm that the large values standard deviations of support stiffness can be one of the important factors causing an intensive dynamic response of the track components.

\section{Conclusions}

The response of a railway track that includes uncertainties in the vertical track supports stiffness subjected to a moving railway vehicle (the locomotive of the type E 499 / 85 t) is solved by the finite element method and time-step integration. The computer program developed can incorporate most rail-track parameters and irregularities of the track structure and is suitable for low and mid frequency analyses of the problem. In this study only the variability of vertical track stiffness is considered to be a random input quantity and the rest input parameters are considered deterministic ones, i.e. the simulation passages of the locomotive E 499 ( 85 t) were made on the track without irregularities.
Monte Carlo simulation was applied for these cases to estimate the dynamic track response to variations of the subgrade stiffness that was simulated as a stationary randomly distributed ballast stiffness with a standard uniform distribution and a normal distribution. The herein presented simulation results of the dynamic interaction are concerned in the low and mid frequencies, say 0 $200 \mathrm{~Hz}$, and they are applied to the track with the stiffness type (B1).

The dynamic amplification resulting from the simulated passage of a vehicle over the simulated track section can be calculated as the ratio of the maximum dynamic response $Y_{d y n}$ (deflection $w$, bending moment $M$, etc.) to the static response $Y_{s t}$ on a given track stiffness level.

Some obtained results can be summarised as follows:

- The dynamic response results due to the tracks with the constant stiffness of supports (deterministic cases) show a low dynamic amplification $\delta=1,05-1,2$ for the track response $Y=(w, P$, $M$, etc.) in relation to the vehicle speed $c$. 
- The dynamic response due to the stationary randomly distributed stiffness of supports $k_{b, j}$ with the standard uniform and normal distribution follows the results of the static analysis and shows how the vehicle speed influences the track response. In these cases the effect of stochastic rail support stiffness can not be ignored, in particular for higher vehicle speeds. While the mean value of the response amplitudes and $\bar{Y}$ the corresponding dynamic coefficients $\delta$ attain no high values for these cases, the individual response amplitudes $Y$ can attain values that can not be ignored. Thus, the expected values of the dynamic coef- ficient $\delta$ may be expected form the histograms of the amplitudes of a quantity $Y_{d y n(\text { rand })}$.

- The standard deviations of support stiffness $\sigma_{k}$ is an important factor affecting the dynamic response on a given level of support stiffness. For example, the maximum dynamic amplitudes of a quantity $Y_{d y n(\text { rand })}$ for the track of the type (A) and (E) can differ nearly by $100 \%$. Generally we can state that the dynamic track response with a low support stiffness is more sensitive than the track with a higher stiffness.

\section{References}

[1] FRÝBA, L.: Vibration of solids and structures under moving load. Academia Praha, 1973.

[2] KNOTHE, K., GRASSIE, S. L.: Modelling of railway track and vehicle/track interaction at high frequencies. Vehicle System Dynamic, $22(1993)$

[3] MORAVČÍK, M.: Experience in Railway Track Testing for Validation of Theoretical Dynamic Analysis, Communications - Scientific Letters of the University of Zilina, EDIS ŽU 1/99.

[4] MORAVČÍK, M.: Vertical track stiffness in service conditions (in Slovak). Technical report for the Slovak Railways. University of Žilina, 1996, 148 p.

[5] MORAVČÍK, M., MORAVČÍK, M.: Track mechanics I (300 p), II 320 p), III 220 p), EDIS Žilina, 2002.

[6] SIČÁR, M.: Vehicle-track interaction concentrated to the track response (in Slovak). PhD Thesis, University of Žilina, 1996, 180 p. 